\section{Review Article}

\section{Corresponding Author}

Andrei Fernandes Joaquim

(iD https://orcid.org/0000-0003-2645-0483

Department of Neurosurgery, State

University of Campinas (UNICAMP),

Campinas, SP, Brazil

Email: andjoaquim@yahoo.com

Received: August 29, 2020

Revised: September 10, 2020

Accepted: September 11, 2020

\title{
Circumferential Operations of the Cervical Spine
}

\author{
Andrei Fernandes Joaquim ${ }^{1}$, Nathan J. Lee ${ }^{2}$, K. Daniel Riew ${ }^{2}$ \\ ${ }^{1}$ Department of Neurosurgery, State University of Campinas (UNICAMP), Campinas, SP, Brazil \\ ${ }^{2}$ Department of Orthopedics, Columbia University, New York, NY, USA
}

Generally, a combined anterior and posterior cervical approach is associated with significant morbidity since it requires an extended operative time, greater intraoperative blood loss, and both anterior- and posterior-related surgical complications. However, there are some instances where a circumferential cervical fusion can be advantageous. Our objective is to discuss the indications for circumferential cervical spine procedures. A narrative review of the literature was performed. We include the indications for circumferential cervical approaches of the senior author (KDR). Indications for circumferential approaches include: (1) high-risk patients for pseudoarthrosis, (2) cervical deformity (e.g., degenerative, posttraumatic, cervicothoracic kyphosis), (3) cervical spine metastases (especially those with multilevel involvement), (4) cervical spine infection, (5) unstable cervical trauma, (6) movement disorders and cerebral palsy, (7) Multiply operated patient (especially postlaminectomy kyphosis and patients with massive ossification of the posterior longitudinal ligament), and when (8) early fusion is desirable. Circumferential procedures may be useful in many different cervical spine conditions requiring surgery. Despite its advantages, particularly with reducing the risk for pseudarthrosis, the benefits of a combined approach must be weighed against the risks associated with a dual approach. With appropriate preoperative planning, intraoperative decision-making, and surgical techniques, excellent clinical outcomes can be achieved.

Keywords: Circumferential, Anterior cervical approach, Posterior cervical approach, Combined cervical approaches

\section{INTRODUCTION}

Described more than 50 years ago by Smith and Robinson, anterior cervical surgery is one of the most common procedures used to stabilize and decompress the cervical spine. ${ }^{1}$ Although many cervical spine diseases can be treated with a posterior cervical approach as well, anterior surgery is generally favored in the setting of kyphosis or when there is significant ventral cord compression. ${ }^{2,3}$ In some patients, there is true equipoise between an anterior and posterior operation, and the route chosen is based on surgeons' and patients' preferences. ${ }^{3}$

However, in some situations, a circumferential approach may be recommended to treat cervical spine conditions, especially those with multicolumn instability and significant risk for pseudarthrosis. ${ }^{4,5}$ Potential benefits include greater potential for de- formity correction, shorter constructs, avoidance of complications associated with instrumentation, nearly guaranteed $100 \%$ fusion rate, avoidance of graft dislodgment that might occur with extensive reconstructions, elimination of an additional anesthetic procedure, and reduction in cost compared to multiple separate procedures. ${ }^{5}$ The potential benefits of a combined approach must be weighed against the potential risk of an additional surgery after a single approach. An additional posterior procedure may result in airway edema due to prolonged operative time, risk of posterior wound complications and infections, and nerve root injuries. For these reasons, a preoperative planning with appropriate patient selection is mandatory. The objective of this paper is to discuss the current literature regarding combined cervical approaches and the senior author (KDR) indications for circumferential cervical spine procedures, as well 
as tips and pearls for practice for surgeons who will perform combined cervical approaches.

\section{METHODS}

We performed a narrative review of the literature on the indications and outcomes for various surgical approaches to the cervical spine. We include a discussion of the indications for circumferential cervical approaches of the senior author (KDR) with illustrative cases and tips for complication avoidance.

Written informed consent was obtained from all the patients presented in this study

\section{RESULTS}

An isolated anterior cervical approach may be appropriate in patients with a relatively low risk for pseudoarthrosis, such as those requiring 1 to 3 level anterior cervical discectomy and fusion (ACDF), 1 level (or even 2 in well-selected patients) ACCF (anterior cervical corpectomy and fusion), and the risk of complications such as graft dislodgment or hardware failure are minimal. ${ }^{3,6}$ In addition, an anterior approach may be favored if there are posterior approach-related concerns (e.g., posterior neck wound issues) or patients with complex medical comorbidities that may preclude a prolonged operative procedure, which is often observed for combined anterior-posterior surgery.

Anterior approaches can be performed for a higher number of fusion levels, but adverse effects significantly increased according to the number of levels treated, especially dysphagia, hardware failure, and pseudoarthrosis. ${ }^{3}$ For instance, pseudoarthrosis rate increases from $2.9 \%$ for 1-level ACDF to $35 \%$ for 3 levels. ${ }^{6}$ The chances of pseudoarthrosis are higher in the caudal levels, especially in long fusions. For these reasons, an isolated anterior approach may be used ideally for decompression and fusion of focal cervical spine disease. ${ }^{3,7,8}$ In this context, we discuss and summarize the main indications for a circumferential fusion of the cervical spine.

\section{High Risk for Pseudarthrosis}

Pseudarthrosis is one of the most common complications after cervical fusion and responsible for nearly half of all revision cervical surgeries. ${ }^{6}$ Subsequent revision surgeries are costly and associated with increased morbidity in comparison to primary cervical fusion. These include higher rates for thromboembolic events, surgical site infections, perioperative blood transfusion, readmission, and return to the operating room. ${ }^{9-11}$ To avoid these complications, a plethora of literature has identified potential risk factors for pseudarthrosis. These include multiple patient-specific factors such as smoking, obesity, diabetes, chronic steroid use, osteoporosis, malnutrition. ${ }^{12,13}$ Some studies even report younger patients are at higher risk for pseudarthrosis due to the relatively higher physical demands as well as poor compliance to physical therapy. Surgical risk factors include a higher number of fusion levels, bone graft type, surgical approach, and type of instrumentation. ${ }^{13}$ Although a combined anterior and posterior fusion construct involves greater operative time and associated with its potential complications, a $360^{\circ}$ fusion construct has been known to support greater mechanical stability necessary for fusion. Several biomechanical studies demonstrate that a $360^{\circ}$ construct provides greater fixation rigidity, particularly with axial rotation. ${ }^{14,15}$ For patients with multiple risk factors and who require multilevel fusion, a combined anterior-posterior cervical fusion can be beneficial since it may provide the necessary mechanical stability for fusion than a single approach (e.g., anterior vs. posterior) can achieve alone. Fig. 1 illustrates a patient with a high risk for pseudoarthrosis.

\section{Cervical Deformity}

A cervical kyphosis is a debilitating form of sagittal deformity that may require surgical correction in patients with severe neck pain, neurological deficits, and functional disabilities. ${ }^{16}$ The etiology is multifactorial (e.g., degenerative disease, trauma, and iatrogenic); however, the major goals remain the same: neural decompression, restoration of cervical and global sagittal alignment, and maintenance of cervical deformity correction with stable fusion. Compared to the posterior approach, an anterior approach can achieve these goals through the direct removal of compressive lesions causing myelopathy and an anterior release via osteotomy for deformity correction. Generally, anterior decompression and fusion is considered effective with acceptable complication rates in these patients. ${ }^{3}$ However, in patients with greater degrees of cervical kyphosis, rigid deformities, and those who require a higher number of levels fused ( $>3$ levels), a combined anterior- and posterior approach may be warranted.

Song et al. ${ }^{17}$ performed a comparative study evaluating the radiographic, clinical, and patient-reported outcomes between anterior fusion and a combined anterior and posterior fusion for degenerative cervical kyphosis with a minimum 2-year follow-up. Both cohorts had similar patient demographics, preoperative kyphotic angles, and breakdown of fixed versus flexible kyphotic patients. Interestingly, the combined cohort under- 

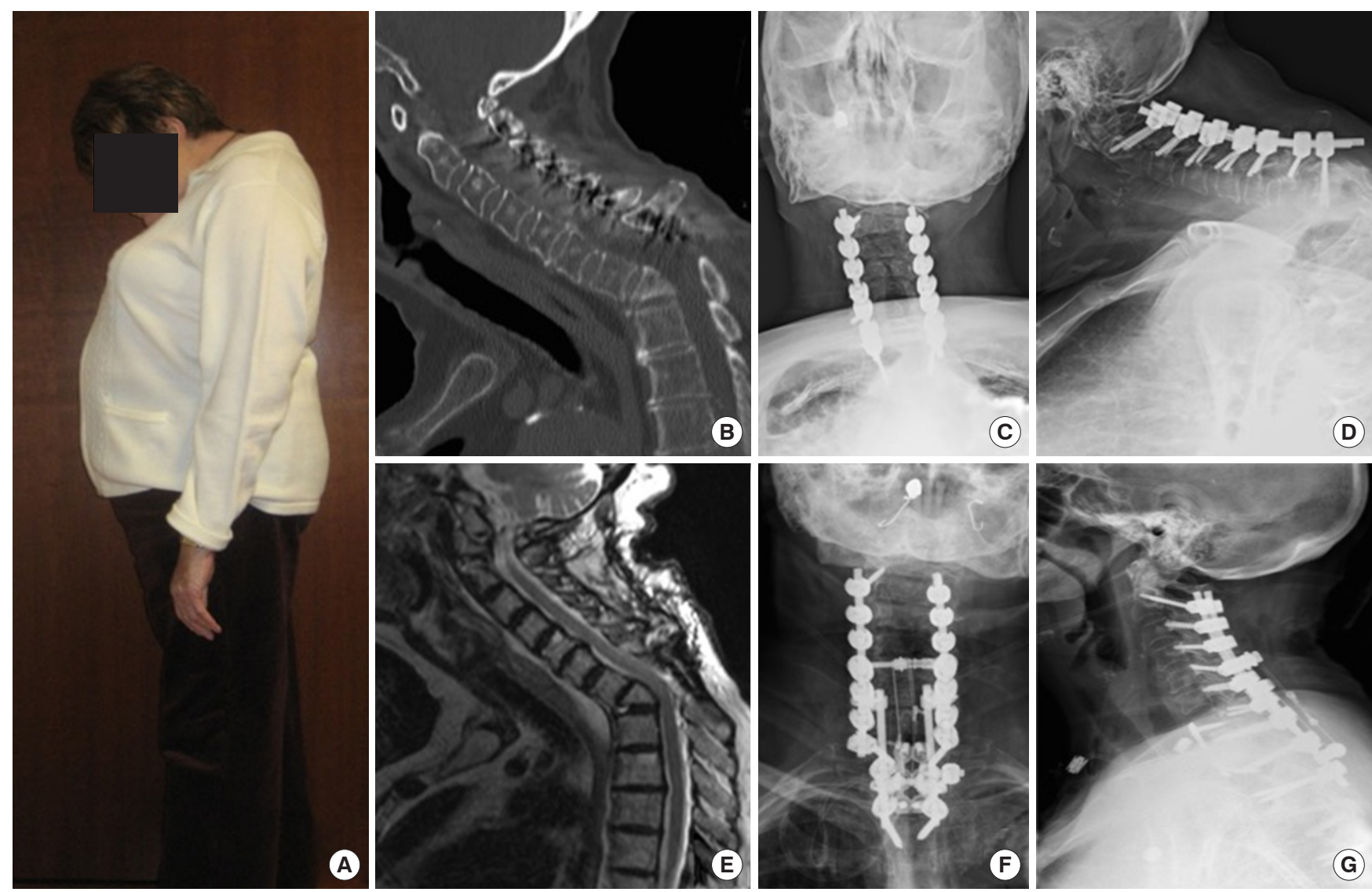

Fig. 1. (A) This 80-year-old lady with chronic obstructive pulmonary disease and chronic use of steroids had a previous C2-T1 fusion for treating a Dropped Head. She developed a cervicothoracic kyphosis due to an insufficiency fracture at T1, with vertebral collapse, as demonstrated on sagittal computed tomography scan (B), anteroposterior (C), and lateral (D) cervical x-rays, and sagittal T2 sequence magnetic resonance imaging (E). She underwent a T1 corpectomy and fusion, followed by revision of the posterior instrumentation, from C2 to T3, as demonstrated in the anteroposterior (F) and lateral ( $\mathrm{G}$ ) postoperative $\mathrm{x}$-rays.

went a higher mean number of fusion levels (mean: 3.6 vs. 2.1). The combined cohort achieved and maintained a higher degree of sagittal correction than did an anterior fusion alone. In terms of patient-reported outcomes, Song et al. ${ }^{17}$ reported that the improvements in pain and neck disability index were significantly better in the combined cohort than the anterior fusion group. It is important to note that the mean operative time and estimated blood loss was nearly 3 -fold greater in the combined fusion group than the anterior group. However, surgical complications were similar or worse in the anterior group. Specifically, the combined cohort experienced significantly less pseudarthrosis, cage subsidence, and hardware-related complications. The mechanism of failure for an isolated anterior fusion in these patients may be related to the excessive load sharing experienced by a construct that is placed too anterior to the axis of rotation of the spine. Any remaining kyphotic alignment will increase the load on the anterior plate in flexion and lead to failure of the correction and pseudoarthrosis. ${ }^{18,19}$

Recently, Smith et al. ${ }^{20}$ presented a prospective, multicenter study on the complication rates after adult cervical deformity surgery with a minimum 1-year follow-up. Consistent with prior literature, the combined group had substantially longer operative time and greater estimated blood loss than either anterior- or posterior fusion alone. However, the complication rates were similar, except dysphagia (combined, $20 \%$; anterior, $17 \%$; posterior, $2 \%$; $\mathrm{p}=0.006$ ).

Given these findings, circumferential cervical fusion, although technically demanding, represents a viable option in the treatment of patients with cervical deformities as this technique promotes greater fusion rates through immediate rigid 3-column stabilization of the cervical spine.

Finally, combined approaches are useful for treating patients with cervicothoracic kyphosis. Anterior cervical discectomies and corpectomies are used to correct the deformity. In some 


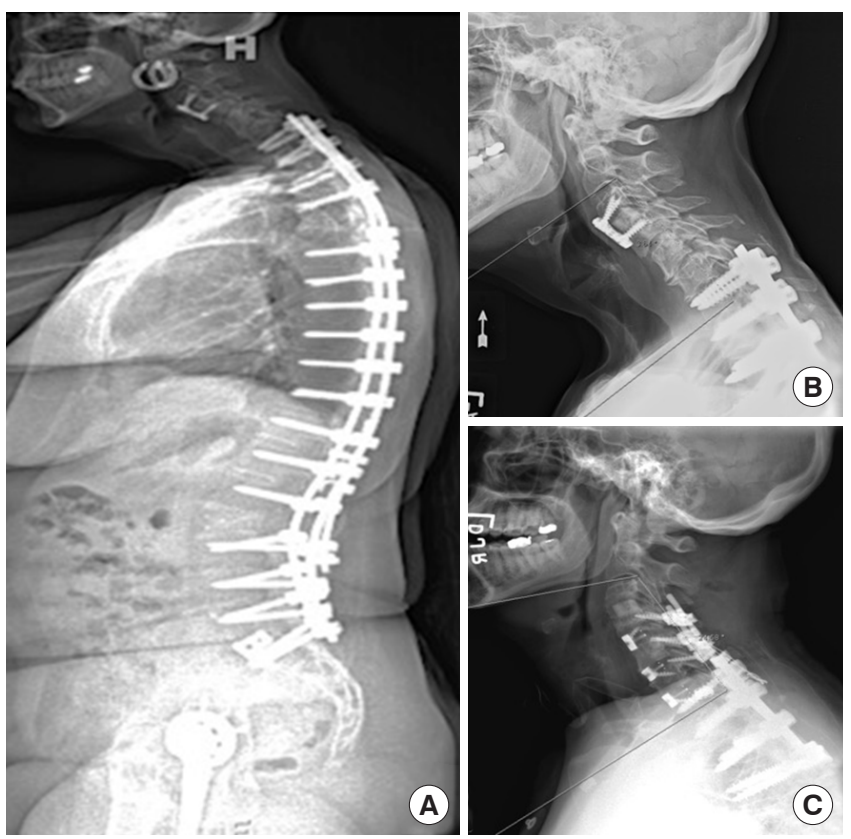

Fig. 2. This 65-year-old woman with severe osteoporosis had C7-S1 posterior fusion, a C4-5 anterior cervical discectomy and fusion, as demonstrated in the long-standing lateral $\mathrm{x}$-ray (A) and lateral cervical $\mathrm{x}$-ray (B). She developed a symptomatic postjunctional kyphosis. A multilevel anterior discectomies and fusion were performed, from $\mathrm{C} 4-7$ followed by posterior C47 supplementation, connecting the cervical rods with the posterior thoracic rods, as demonstrated in the postoperative cervical lateral $\mathrm{x}$-ray $(\mathrm{C})$.

cases, this may be extended to a median sternotomy followed by a posterior approach that would restore the posterior tension band. ${ }^{16}$ Initially, anterior approaches were able to restore cervicothoracic kyphosis in flexible deformities. However, the anterior osteotomy, described by the senior author, allowed corrections of rigid deformities by an anterior approach, consisting of an osteotomy through the cervical disc and uncovertebral joints, placement of initially divergent Caspar pins that will produce lordosis once the distractors are placed. A vertebral body spreader and pushing on the head may provide a further correction. ${ }^{5}$ This technique provides excellent angular and sagittal correction, followed by a posterior supplementation, being an option for posterior-anterior-posterior approaches used in the cases of posterior arthrodesis or spinal ankylosis. Fig. 2 illustrates a 65-yearold woman who had cervical deformity treated with a circumferential cervical surgery.

\section{Cervical Spine Metastases}

Metastatic disease of the cervical spine often necessitates surgery to address the onset of neurological deficits and instability.
In these cases, surgery is often palliative due to the relatively shorter life expectancy in these patients but can still improve their pain and function to a considerable degree. ${ }^{21}$ Fehlings et al. ${ }^{22}$ performed an elegant qualitative systematic review of the literature about the decision-making in the surgical treatment of cervical spine metastases. For both the subaxial spine (C3-6) and the cervicothoracic junction (C7-T2), they recommend a combined anteroposterior approach when there is radiographic involvement of all 3 columns, multilevel disease ( 2 or more levels), and poor bone quality. Especially for the cervicothoracic junction (C7-T2), higher failure rates have been reported for anterior stand-alone procedures. ${ }^{23,24}$ Similarly, biomechanical studies have shown that posterior-alone constructs do not provide adequate fixation stiffness for 3-column instability. ${ }^{25,26}$ It is important to note that these recommendations, although strongly supported by the authors, were not based on any level-1 or level-2 studies. Another important consideration is that many oncological patients are on steroids, require radiation therapy, and have relatively poorer bone quality, which increase the risk for implant failure and pseudarthrosis. A revision surgery would be particularly detrimental for these patients as it would likely require a lengthier hospital stay and compromise their already tenuous life expectancy. Fig. 3 illustrates a case of a metastatic tumor at $\mathrm{T} 1$ treated with a combined approach.

\section{Cervical Spine Infection}

In severe cervical osteomyelitis cases where multiple levels and columns of the spine are involved, a combined approach is more advantageous than a single approach alone. The anterior approach can effectively expose the diseased vertebrae under direct vision and remove necrotic debris causing spinal cord compression. However, after anterior debridement, placement of multilevel internal fixation can be difficult, especially for caudal levels of the cervical spine. When an infectious lesion destructs the cervicothoracic junction, kyphosis and collapse can easily form, and an anterior approach has not been successful in preventing the progression of kyphosis or corrective preexisting kyphosis, especially when there are multiple levels involved. $24,27,28$

Zhang et al..$^{29}$ reported successful treatment of 15 patients with cervicothoracic kyphosis secondary to tuberculosis operated with circumferential approaches, with fusion in all cases, as well as infection control and neurological improvement. The procedures were performed from C6 to T3, consisted of posterior fixation, anterior debridement, bone grafting, and anterior fixation. There was a significant improvement in the kyphosis angle 

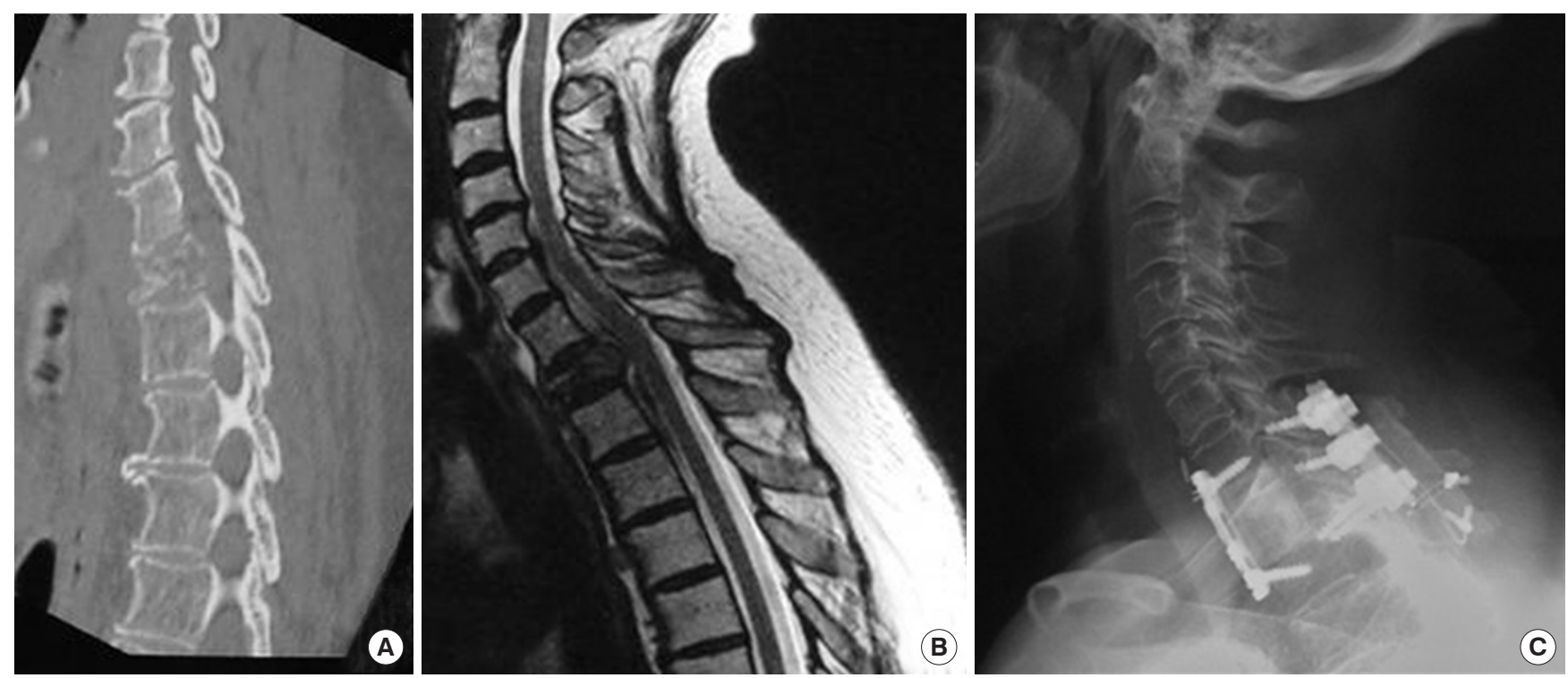

Fig. 3. This 71-year-old lady with a breast cancer metastatic tumor at T1 with spinal cord compression, with vertebral T1 collapse at sagittal computed tomography scan (A) and spinal cord compression at T2 sequence sagittal magnetic resonance imaging (B), underwent a T1 corpectomy, followed by a posterior C7-T12 fusion, with immediate stability as shown in lateral cervical plain radiograph $(\mathrm{C})$.

$(\mathrm{p}<0.05)$ after an average follow-up of 27.7 months, suggesting durable results, without surgical complications.

Although instrumentation is known to improve fusion rates, there is a concern for bacterial seeding of hardware in the setting of an active infection. In a systematic review by Wang et al., ${ }^{30}$ the incidence of hardware failure (defined as screw loosening, pull-out, graft subsidence) and wound complications (defined as reoperations requiring wound debridement and/or removal of hardware because of persistent infection) after circumferential fixation was comparable to those for noninfectious elective cases.

\section{Unstable Cervical Trauma}

Cervical spine injuries with severe instability can safely and more effectively be treated with a combined anterior and posterior fusion. Cervical instability can result when there is bony and/or soft tissue compromise through multiple columns of the cervical spine. For instance, a flexion-distraction injury can result in disruption of posterior spinal ligaments, facet joints, longitudinal ligaments, and annulus fibrosis. When there is severe instability, such as bilateral facet dislocations or fractures involving both facets, an anterior fixation alone is less effective in achieving adequate fusion and stability. ${ }^{31-34}$ Stauffer et al. ${ }^{35}$ and Tucker ${ }^{36}$ reported kyphotic deformity and graft extrusion after anterior surgery alone for cervical spine trauma, potentially due to severe posterior ligamentous injury and failure anterior col- umn support. For lower cervical spine injuries, an anterior approach alone can result in postoperative kyphosis without appropriate posterior fixation support. A posterior-alone approach can address facet dislocations and posterior fractures such as a lamina fragment in the canal; however, when the anterior column is involved and restoration of cervical lordosis is necessary, a posterior-alone approach is not commonly used, despite it is also an option..$^{37}$ In comparison, a combined approach provides superior internal fixation, higher bony fusion rates, the ability to restore and maintain cervical alignment, and directly decompress compromised neural structures. ${ }^{38-40}$ Furthermore, McAfee et al. ${ }^{41}$ reported a higher rate of successful outcomes when considering neurological improvement and complications after circumferential approach for cervical trauma in 31 patients, suggesting that it may save levels of fusion and provide robust and durable results. For completely dislocated hangman's fractures with injuries to the $\mathrm{C} 2-3$ disc and anterior longitudinal ligament, a posterior approach cannot directly decompress the spinal cord compression arising anteriorly and requires $360^{\circ}$ fixation to restore biomechanical stability of the upper cervical spine. In a case series of 11 patients with completely displaced hangman's fractures who underwent combined anterior-posterior fusion, Wang et al. ${ }^{42}$ found that this procedure provides good neurological recovery of function and achieves solid fusion with an acceptable risk of complications. 


\section{Movement Disorders (Parkinson, Dystonia, etc.) and Cerebral Palsy}

Movement disorders are associated with severe degenerative spinal diseases. For instance, dystonic patients have a high rate of upper cervical spine level degeneration (C2-5) when compared with nondystonic patients with cervical spondylotic myelopathy. ${ }^{43}$ Surgery is complicated in these patients due to the higher risk of spinal instability for a poorly controlled disorder. Additionally, the prevalence of degenerative changes in patients with movement disorders is higher than in the general populations: more than $50 \%$ of adult patients with athetoid cerebral palsy had severe degenerative changes in radiological exams. ${ }^{44}$

For patients with movement disorders, circumferential stabilization is highly recommended as the procedure of choice due to higher fusion rates and better potential for decompression and reconstruction, especially in multilevel procedures. ${ }^{43}$ In one of the largest series of patients with athetoid cerebral palsy, 23 cases reported successful outcomes after a combined anteroposterior approach with a mean follow-up of 8.7 years. At 1-year follow-up, 12 patients had ambulation improvement and strength improved in almost all patients, even in those with severe involuntary movements. ${ }^{45}$ In the same context, Parkinson patients are at high risk for pseudoarthrosis and complications after spinal procedures, with less satisfactory outcomes when compared to non-Parkinson patients. ${ }^{46}$ For these reasons, a circumferential fusion, which provided early immobilization and rigid fixation necessary for bony fusion, is highly recommended for these high-risk patients.

\section{Multiple Cervical Surgeries}

A corpectomy requires an anterior plate to prevent graft extrusion, which is a complication that is exponentially more common when longer strut grafts are used for multilevel corpectomies. For these patients, supplemental posterior fixation is needed. ${ }^{3}$ An alternative is to use a buttress plate that is only fixed at one end of the construct to avoid graft extrusion until a posterior fixation is performed. However, a short buttress plate fixed with only 2 screws should never be used following a corpectomy, as a kick-out of the plate can cause catastrophic airway compromise and death. ${ }^{47}$ Without posterior instrumentation in these cases, a long strut migration can result in catastrophic airway obstruction and death. For 2 or more level corpectomies, we strongly recommend anterior plating followed by posterior supplementation to minimize the risk of graft extrusion, although a 2-level corpectomy with plating may be performed isolated in well-selected patients (without risk factor for pseudoarthrosis).
Postlaminectomy kyphosis is an iatrogenic complication after surgery for several cervical pathologies, including spinal tumors, cervical spondylotic myelopathy, and infection. The posterior column disruption in combination with a ventral corpectomy leads to a highly unstable spine. Riew et al. ${ }^{48}$ demonstrated a high rate of early graft-related complications in 9 of 16 postlaminectomy patients (56\%), who underwent multilevel anterior cervical corpectomy, with 4 of them requiring additional surgery. A total of 3 of the 4 graft extrusions occurred during halo immobilization. For these reasons, posterior stabilization is strongly recommended after multilevel corpectomies or after an extensive anterior approach required to restore lordosis after a postlaminectomy kyphosis.

When considering multiple cervical spine surgeries, we use the following aphorism as guidance: the second operation must be the last one - we do not want a third procedure. In this context, circumferential fusions may be a very reasonable option for definitive cervical stabilization.

A postlaminectomy deformity corrected with a circumferential approach is illustrated in Fig. 4.

\section{Early Fusion Desirable}

There is a consensus in the literature that professional and elite athletes can safely return to collision and high-energy sports after single-level subaxial ACDF with modern anterior plating techniques. However, patients requiring faster healing and fusion to return to physically-demanding jobs such as construction work earlier might consider a circumferential approach for multilevel procedures. Other considerations for earlier fusion include patients with relatively poor compliance or international patients who may not return for follow-up care or additional surgeries due to travel constraints. In these cases, early fusion is necessary and most likely to be achieved with a circumferential approach.

\section{POTENTIAL RISKS ASSOCIATED WITH COMBINED PROCEDURES THAT SHOULD BE TAKEN IN INTO ACCOUNT FOR SURGICAL PLANNING}

\section{Root Injuries}

Posterior cervical fusion alone may add a risk of postoperative root deficits. These have been reported for $\mathrm{C} 5, \mathrm{C} 8$, and $\mathrm{T} 1$ nerve roots. ${ }^{48} \mathrm{C} 5$ palsy has many definitions, but generally is characterized by a deltoid and (usually) biceps muscle weakness after surgery, generally unilateral (in more than $90 \%$ of the cas- 

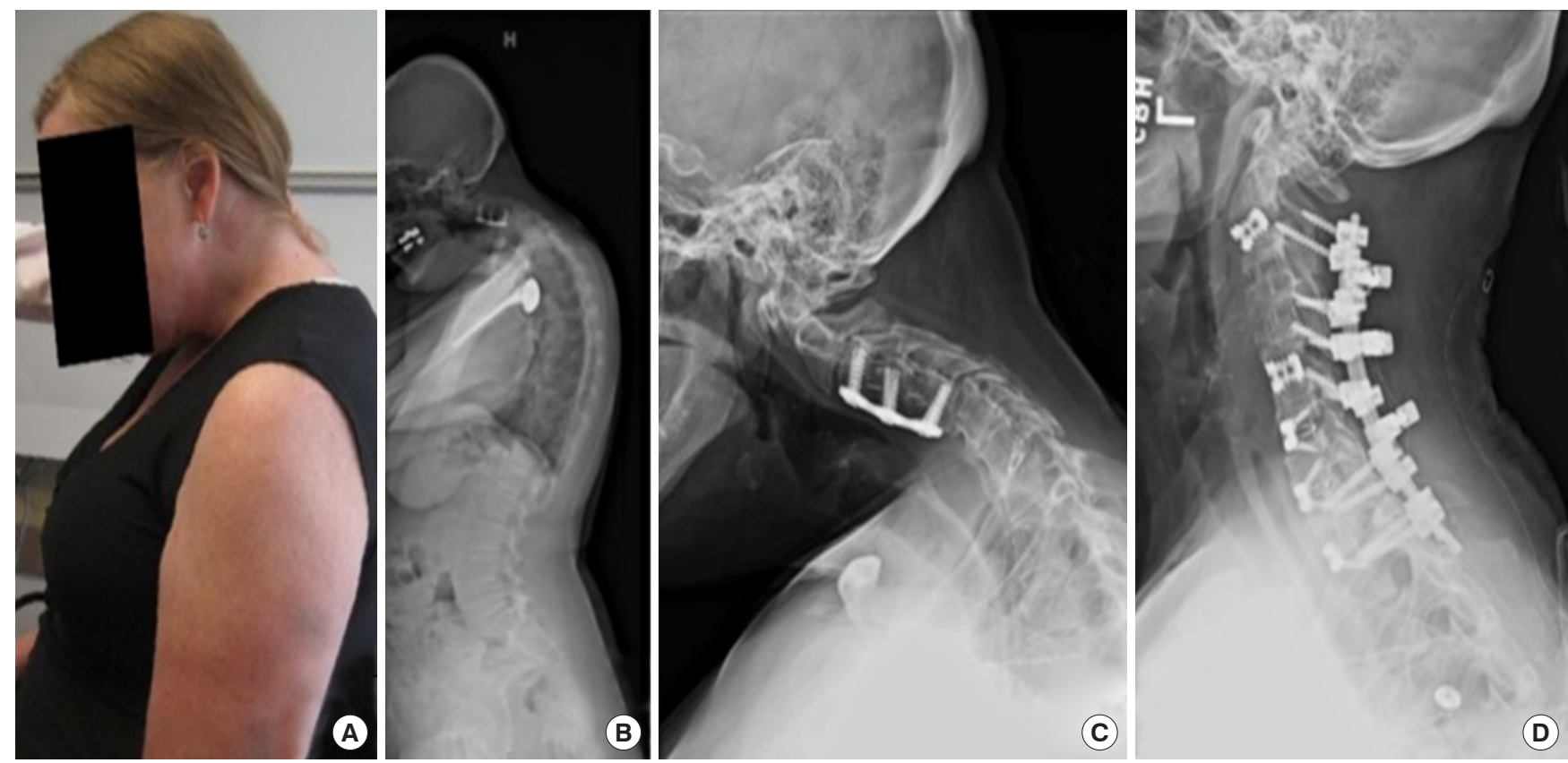

Fig. 4. This 51-year-old lady (A) had a previous 2-level anterior cervical discectomy (C3-5) and fusion with a postlaminectomy kyphosis as shown in lateral $\mathrm{x}$-rays (B, C). She was an opioid-dependent and recent smoker. She had plate removal, multilevel anterior cervical discectomy and fusion (C2-3, C5-6, C6-7, T1-2) followed by a C2-T2 fusion, with good correction (D).

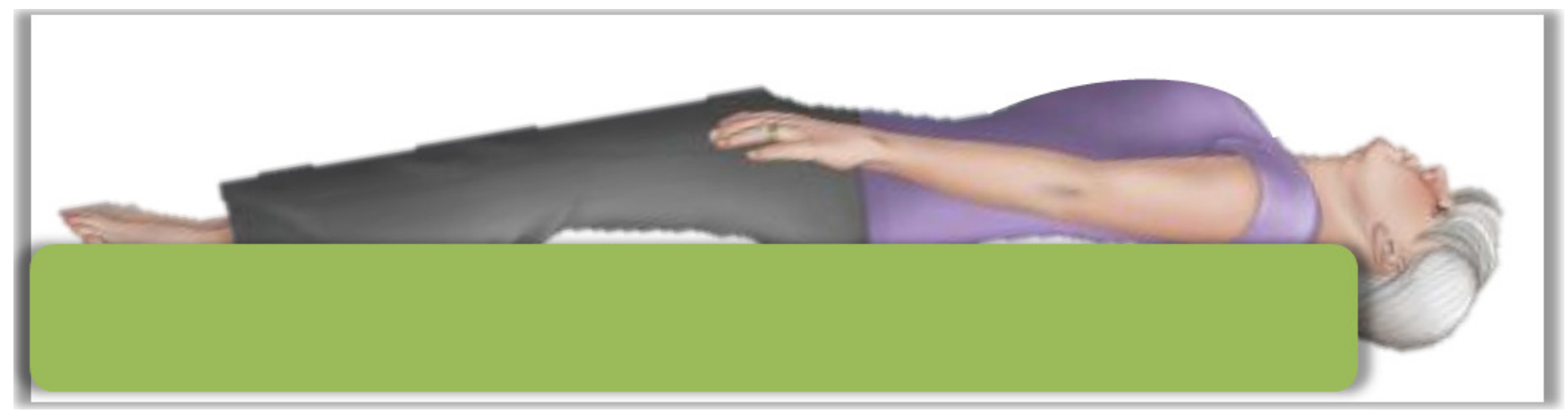

Fig. 5. Preoperative lordosis position test-the patient is placed in a supine position with the neck extended for several minutesif the patient develops paresthesia, weakness, or pain in the arms, an anterior approach at the stenotic levels or a posterior foraminotomy is necessary during a posterior surgery to avoid postoperative $\mathrm{C} 8$ and $\mathrm{T} 1$ deficits.

es). ${ }^{49,50}$ It occurs more commonly after posterior cervical procedures-potentially explained by its vulnerability to traction with the translation of the spinal cord posteriorly with a horizontal and shorter trajectory of the roots. For this reason, an anterior C4-5 discectomy may increase foraminal height, which may decrease the risk of $\mathrm{C} 5$ palsy after an isolated posterior approach.

C8 and T1 nerve palsies are relatively rare compared to other nerve root injuries and characterized by weakness of all intrinsic muscles of the hand, numbness in the fourth and fifth fingers as well as the ulnar border of the hand and forearm. ${ }^{49} \mathrm{To}$ avoid postoperative $\mathrm{C} 8$ and $\mathrm{T} 1$ deficits, we can perform a preoperative lordosis position test (Fig. 5). It involves placing the patient in a supine position with the neck extended for several minutes: if the patient develops paresthesia, weakness, or pain in the arms, an anterior approach at the stenotic levels or a posterior foraminotomy during the posterior approach is necessary. However, when there is significant disc height loss at the level of the foraminal stenosis, a foraminotomy-alone may not fully address the stenotic level (Fig. 6). This is because in patients with a collapsed disc, the interpedicular foraminal height is narrowed. Placing such a level in lordosis further shortens this 

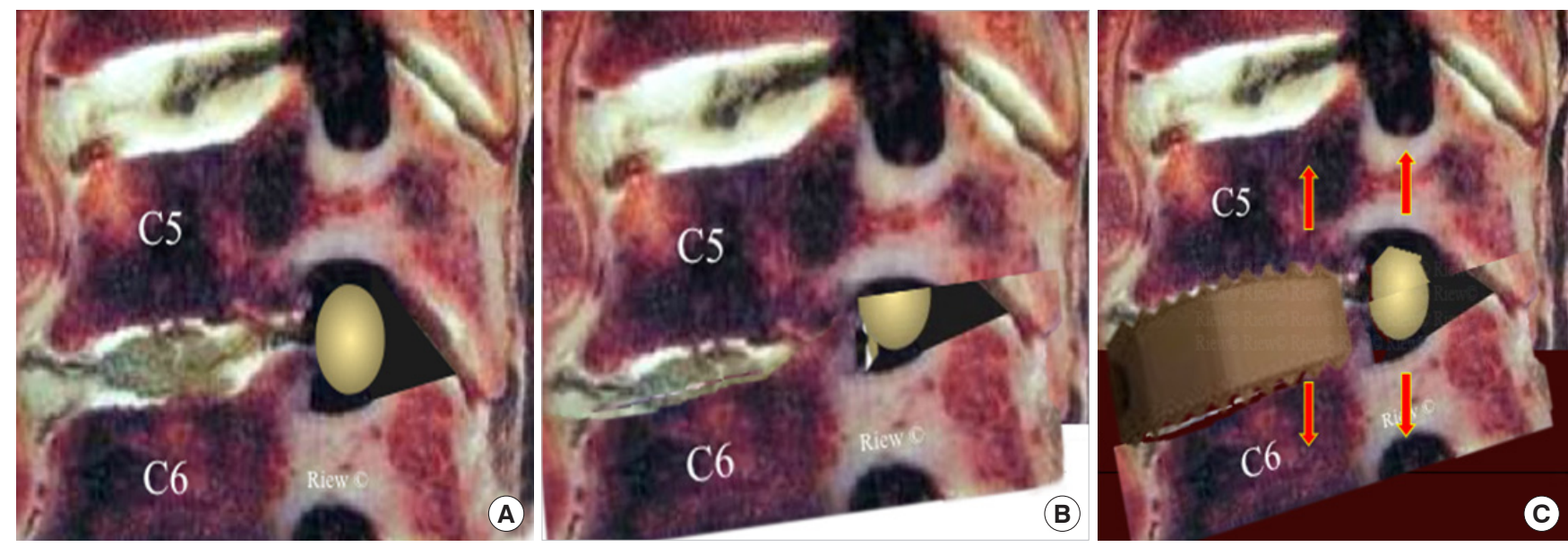

Fig. 6. Illustrative pictures of the foraminal height at the level of C5-6. When there is some disc height loss (A) at the level of foraminal stenosis, a foraminotomy-alone will not fully address cranial-caudal stenosis, once extension for deformity correction (B) will cause compression due to decrease foraminal height. In such cases, restoration of disc height via an anterior approach (C) is needed prior to cervical extension and a posterior foraminotomy. Red arrows represent vertical increase of the cervical foramen.

height. A foraminotomy increases the anteroposterior dimension of the foramen but not its height. Restoration of disc height through an anterior approach first can distract the stenotic foramen. According to the senior author, about $90 \%$ of correction is made anteriorly, using stand-alone devices with one screw that may allow further posterior correction. Restoring disc height will improve the foraminal height and thereby decreases the chances of nerve root injury during and after surgery.

\section{Airway Compromise}

This is one of the most devastating complications after anterior cervical surgery and most commonly the result of cervical hematoma and/or airway edema. ${ }^{3,51}$ A combined approach requires a longer operative time, which increases the risk for retraction-related injuries, swelling, and bleeding. To help decrease the risk of retropharyngeal edema, we place the patient in a reverse Trendelenburg position throughout the entire posterior procedure (Fig. 7). Meticulous hemostasis is mandatory to avoid hematoma, which requires a good surgical inspection prior to closure. The senior author wraps the edges of the longus colli with Surgicel, which is an absorbable hemostatic agent to further control postsurgical bleeding. ${ }^{3}$ A $1 / 4$-inch Penrose drain is left in place to minimize the accumulation of hematoma or seroma (Fig. 8). The senior author has tried multiple types of drains and a Penrose is the only one that has never clotted off. Of note, Penrose drains are routinely used by many ENT (ear, nose, and throat) surgeons and have been used by many cervical spine surgeons, dating back to Dr. Robbie Robinson, who described

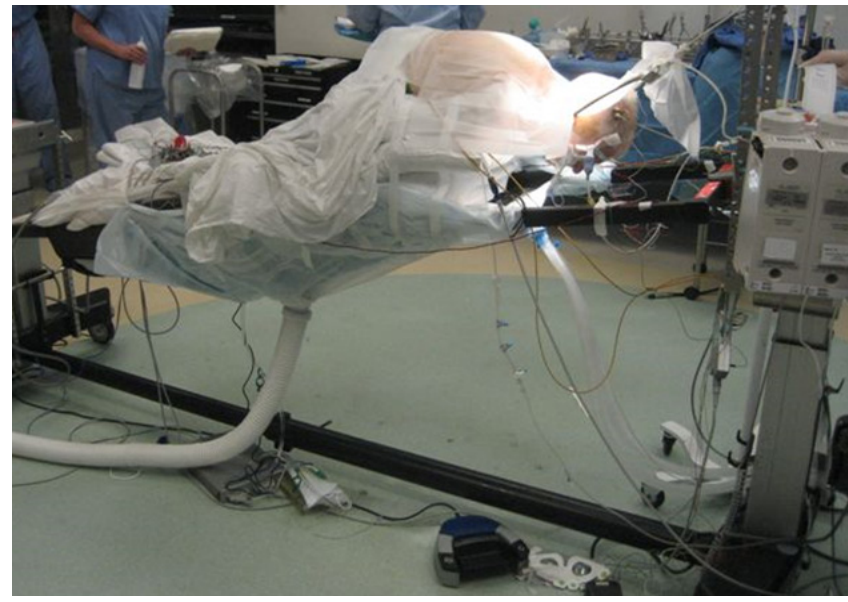

Fig. 7. Illustrative view of the patient position - a reverse Trendelenburg position is preferred to reduce retropharyngeal edema. Intermittent pneumatic stocking to prevent deep vein thrombosis is required.

the Smith-Robinson approach to the cervical spine. The senior author has used Penrose drains throughout his 25-year career and has had a very low infection rate compared to the reported rates in the literature. We believe that, similar to leaving an infected wound open to allow for drainage, the Penrose drain keeps the wound open. Because the lumen is large and does not rely on suction, it is less prone to clotting off than closed suction drains. We have had retropharyngeal hematomas even with closed suction drainage, as they tend to clot if there is significant bleeding. However, with a Penrose drain, we have not had a retropharyngeal hematoma, even with heavy drainage. 

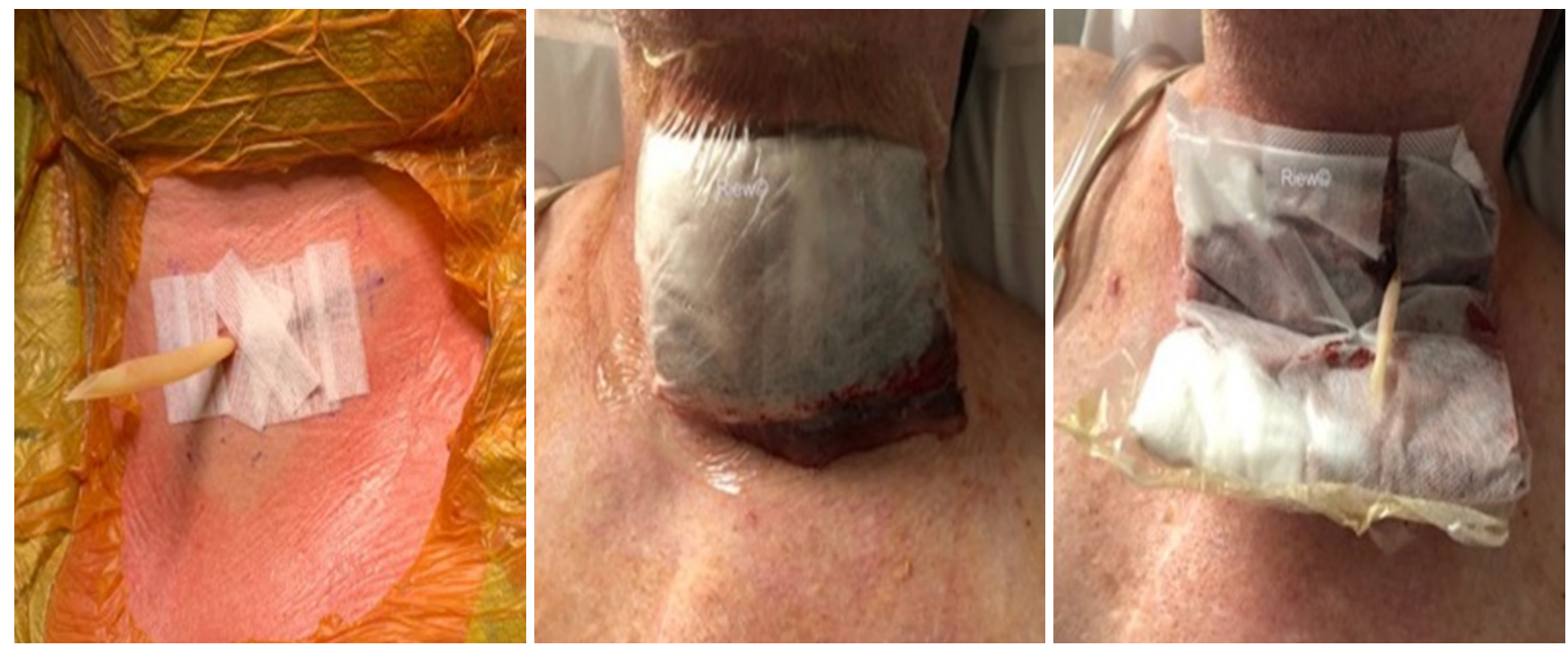

Fig. 8. The senior author prefers to use a 1/4-inch Penrose drain after anterior cervical surgery. This involves supported by steristrips about the wound, an abdominal pad, and transparent adhesive, as illustrated in the 3 pictures above.

Retropharyngeal steroids have been shown to reduce the risk of dysphagia in a number of studies. In a prospective, randomized study, Lee et al. ${ }^{52}$ found that retropharyngeal steroid use significantly reduced prevertebral soft tissue swelling and dysphagia compared to those who did not receive steroids. In addition, steroid use may be particularly beneficial when bone morphogenetic proteins are used and in combined approaches. However, care must be taken with retropharyngeal steroid use. Lee et al. ${ }^{53}$ reported on 2 cases of delayed esophageal perforation, potentially attributed to retropharyngeal corticoids use that required surgical repair and antibiotics, which should be considered as a potentially severe adverse effect of steroids. We recommend against using any retropharyngeal steroids if there is prominent instrumentation, as it can further erode an esophagus that may be thinned by steroids.

After any cervical surgery, a number of factors should be considered when deciding between immediate extubation after surgery or transfer to the intensive care unit with delayed extubation. Perhaps the most important factor is the relative operative duration of surgery. We use the following criteria. If the retraction time of an anterior approach is less than 3 hours and the posterior approach operative duration is less than 6 hours, then the patient can be extubated. If a longer operative duration is required for either the anterior or posterior approach, we consider keeping the patient intubated overnight with an elective extubation the next day. The operative duration should be considered relative to the number of levels involved. For example, a 1-level surgery requiring 3 hours of cervical retraction will like- ly have much more airway edema postoperatively than 3 hours of cervical retraction for a 5-level ACDF. In the latter case, a shorter time of retraction is spent per level and the more extensive exposure allows for reduced retraction force to expose the spine, thereby decreasing the risk of postoperative retropharyngeal edema. Another consideration for keeping the patient intubated overnight has to do with the time. If the operation finishes late in the evening, it may be safer to keep the patient intubated, since there may be fewer staff to emergently reintubate the patient, should that become necessary.

Prior to extubating a patient who has been kept intubated overnight for airway protection, a cuff leak test (CLT) should be performed. This can be performed as follows: the endotracheal cuff is deflated, and the lumen of the tube is occluded with the finger. If there is no edema, the patient can breathe around the outside of the tube. On the other hand, if edema encloses all of the dead space outside of the tube, there can be no airflow lateral to the tube; the patient fails the test, and intubation should be maintained for 24 to 48 hours to allow time for the airway edema to resolve.

Fontes et al..$^{54}$ performed a prospective observational study in 20 patients who had circumferential cervical spine surgery to analyze the temporal evolution of the CLT and perioperative factors that may require extubation. The institutional protocol consisted of maintaining intubation overnight following surgery and extubation if the CLT was $>200 \mathrm{~mL}$, and both intensive care unit and neurosurgery teams agreed that it was safe. The CLT was measured as it follows: after the endotracheal tube 
was deflated, 2 additional respiratory cycles were performed (to allow coughing) and the expired tidal volume was recorded over the following 6 respiratory cycles - then the cuff was reinflated and ventilator parameters returned to baseline. A quantitative cuff leak was calculated as the difference between the mechanical inspired volume and the average of the 3 lowest tidal volumes with the cuff deflated. The authors also evaluated the luminal area of the infraglottic space using preoperative computed tomography or ma gnetic resonance imaging. Eight patients were extubated on the first postoperative day (early group), and 12 patients had late extubation (delayed group). They reported that the main indication for surgery was a cervical deformity, and patients were intubated by an average of 73.6 hours (range, 26-222 hours). Four patients failed extubation with 3 (15\%) requiring tracheostomy. They reported that patients with larger preoperative cuff leak values tended to have a shorter intubation period $(\mathrm{p}=0.53)$, and the infraglottic luminal area differed significantly between the groups $(\mathrm{p}<0.05)$. They concluded that local anatomic factors might also influence airway outcome in large cervical spine procedures - patients with larger infraglottic luminal areas may benefit from a less strict airway management protocol.

\section{Pseudoarthrosis}

The highest risk of pseudoarthrosis is generally at the bottom of a multilevel anterior-alone construct. This may be clinically relevant in some contexts: for instance, in a multilevel anterior procedure, stand-alone interbody devices with self-fixation screws may be used in the upper levels, preferentially plating the bottom levels. Another clinical relevance is that in a patient who needs a multilevel ACDF, one can opt for immediate circumferential arthrodesis or wait to see if a pseudoarthrosis develops. Since pseudoarthrosis usually develops at the bottom 1-2 levels only, the revision posteriorly procedure will often be a minor procedure.

\section{CONCLUSIONS}

Circumferential cervical fusion is a technically demanding surgery that is known to be associated with prolonged operative time, greater intraoperative blood loss, and approach-related complications. However, several indications have been described where a combined approach is advantageous, particularly for those with pathologies that have resulted in multilevel, multicolumn instability, and higher risk for pseudarthrosis that require immediate rigid fixation. In the appropriate setting, a com- bined approach has proven to be a relatively safe and effective surgery compared to single-approach surgeries. With appropriate preoperative planning, intraoperative decision making (e.g., patient positioning), surgical techniques (e.g., thorough decompression, anterior plating of bottom levels, meticulous dissection, and hemostasis), excellent clinical outcomes can be achieved.

\section{CONFLICT OF INTEREST}

The authors have nothing to disclose.

\section{REFERENCES}

1. Robinson RA, Smith G. Anterolateral cervical disk removal and interbody fusion for cervical disk syndrome. Bull Johns Hopkins Hosp 1955;96:223-4.

2. Joaquim AF, Ghizoni E, Tedeschi H, et al. Management of degenerative cervical myelopathy - an update. Rev Assoc Med Bras (1992) 2016;62:886-94.

3. Joaquim AF, Sielatycki JA, Riew KD. Anterior surgical options for cervical myelopathy. Indian Spine J 2019;2:33-41.

4. Zdeblick TA, Hughes SS, Riew KD, et al. Failed anterior cervical discectomy and arthrodesis: analysis and treatment of thirty-five patients. J Bone Joint Surg 1997;79:523-32.

5. Kim HJ, Piyaskulkaew C, Riew KD. Anterior cervical osteotomy for fixed cervical deformities. Spine (Phila Pa 1976) 2014;39:1751-7.

6. Fraser JF, Hartl R. Anterior approaches to fusion of the cervical spine: a meta-analysis of fusion rates. J Neuros Spine 2007;6:298-303.

7. Tan LA, Riew KD, Traynelis VC. Cervical spine deformitypart 2: management algorithm and anterior techniques. Neurosurgery 2017;81:561-7.

8. Tan LA, Riew KD, Traynelis VC. Cervical spine deformitypart 3: posterior techniques, clinical outcome, and complications. Neurosurgery 2017;81:893-8.

9. Kim EJ, Chotai S, Wick JB, et al. Patient-reported outcomes and costs associated with revision surgery for degenerative cervical spine diseases. Spine (Phila Pa 1976) 2018;43:E423-9.

10. Malik AT, Khan SN, Kim J, et al. 30-Day postoperative morbidity and readmission following revision anterior cervical discectomy and fusion (ACDF). Clin Spine Surg 2019;32:435-8.

11. Basques BA, Ondeck NT, Geiger EJ, et al. Differences in shortterm outcomes between primary and revision anterior cervical discectomy and fusion. Spine (Phila Pa 1976) 2017;42: 253-60. 
12. Hofler RC, Swong K, Martin B, et al. Risk of pseudoarthrosis after spinal fusion: analysis from the healthcare cost and utilization project. World Neurosurg 2018;120:e194-202.

13. Leven D, Cho SK. Pseudarthrosis of the cervical spine: risk factors, diagnosis and management. Asian Spine J 2016;10: 776-86.

14. Koller H, Schmidt R, Mayer M, et al. The stabilizing potential of anterior, posterior and combined techniques for the reconstruction of a 2-level cervical corpectomy model: biomechanical study and first results of ATPS Protyping. Eur Spine J 2010;19:2137-48.

15. Koller H, Schmoelz W, Zenner J, et al. Construct stability of an instrumented 2-level cervical corpectomy model following fatigue testing: biomechanical comparison of circumferential antero-posterior instrumentation versus a novel anterior-only transpedicular screw-plate fixation technique. Eur Spine J 2015;24:2848-56.

16. Mummaneni PV, Deutsch H, Mummaneni VP. Cervicothoracic kyphosis. Neurosurg Clin N Am 2006;17:277-87.

17. Song KJ, Johnson JS, Choi BR, et al. Anterior fusion alone compared with combined anterior and posterior fusion for the treatment of degenerative cervical kyphosis. J Bone Joint Surg Br 2010;92:1548-52.

18. O'Shaughnessy BA, Liu JC, Hsieh PC, et al. Surgical treatment of fixed cervical kyphosis with myelopathy. Spine (Phila Pa 1976) 2008;33:771-8.

19. Wang M, Gourab K, McGrady LM, et al. Alteration of load sharing of anterior cervical implants with change in cervical sagittal alignment. Med Eng Phys 2008;30:768-73.

20. Smith JS, Buell TJ, Shaffrey CI, et al. Prospective multicenter assessment of complication rates associated with adult cervical deformity surgery in 133 patients with minimum 1-year follow-up. J Neurosurg Spine 2020 Jun 19;1-13 [Epub]. https: //doi.org/10.3171/2020.4.SPINE20213.

21. Joaquim AF, Powers A, Laufer I, et al. An update in the management of spinal metastases. Arq Neuropsiquiatr 2015;73: 795-802.

22. Fehlings MG, David KS, Vialle L, et al. Decision making in the surgical treatment of cervical spine metastases. Spine (Phila Pa 1976) 2009;34:S108-17.

23. Le H, Balabhadra R, Park J, et al. Surgical treatment of tumors involving the cervicothoracic junction. Neurosurg Focus 2003;15:E3.

24. Boockvar JA, Philips MF, Telfeian AE, et al. Results and risk factors for anterior cervicothoracic junction surgery. J Neurosurg 2001;94:12-7.
25. Kreshak JL, Kim DH, Lindsey DP, et al. Posterior stabilization at the cervicothoracic junction: a biomechanical study. Spine (Phila Pa 1976) 2002;27:2763-70.

26. Prybis BG, Tortolani PJ, Hu N, et al. A comparative biomechanical analysis of spinal instability and instrumentation of the cervicothoracic junction: an in vitro human cadaveric model. J Spinal Disord Tech 2007;20:233-8.

27. Rajasekaran S, Soundarapandian S. Progression of kyphosis in tuberculosis of the spine treated by anterior arthrodesis. J Bone Joint Surg Am 1989;71:1314-23.

28. Fountain SS, Hsu LC, Yau AC, et al. Progressive kyphosis following solid anterior spine fusion in children with tuberculosis of the spine. A long-term study. J Bone Joint Surg Am 1975;57:1104-7.

29. Zhang HQ, Hu X, Yin X, et al. One-stage combined anterior-posterior approach treatment of multiple cervicothoracic spinal tuberculosis with kyphosis. Int Orthop 2015;39:160510.

30. Wang AJ, Huang KT, Smith TR, et al. Cervical spine osteomyelitis: a systematic review of instrumented fusion in the modern era. World Neurosurg 2018;120:e562-72.

31. Henriques T, Olerud C, Bergman A, et al. Distractive flexion injuries of the subaxial cervical spine treated with anterior plate alone. J Spinal Disord Tech 2004;17:1-7.

32. Song KJ, Lee KB. Anterior versus combined anterior and posterior fixation/fusion in the treatment of distraction-flexion injury in the lower cervical spine. J Clin Neurosci 2008;15: 36-42.

33. Vaccaro AR, Madigan L, Schweitzer ME, et al. Magnetic resonance imaging analysis of soft tissue disruption after flexion-distraction injuries of the subaxial cervical spine. Spine (Phila Pa 1976) 2001;26:1866-72.

34. Pitzen T, Lane C, Goertzen D, et al. Anterior cervical plate fixation: biomechanical effectiveness as a function of posterior element injury. J Neurosurg 2003;99:84-90.

35. Stauffer ES, Kelly EG. Fracture dislocations of the cervical spine: instability and recurrent deformity following treatment by anterior interbody fusion. J Bone Joint Surg (Am) 1977; 59A:45-8.

36. Tucker HH. Technical report: method of fixation of subluxed or dislocated cervical spine below C1-C2. Can J Neurol Sci 1975;2:381-2.

37. Park JH, Roh SW, Rhim SC. A single-stage posterior approach with open reduction and pedicle screw fixation in subaxial cervical facet dislocations. J Neurosurg Spine 2015;23: $35-41$. 
38. Miao DC, Wang F, Shen Y. Immediate reduction under general anesthesia and combined anterior and posterior fusion in the treatment of distraction-flexion injury in the lower cervical spine. J Orthop Surg Res 2018;13:126.

39. Shen Y, Shen HL, Feng ML, et al. Immediate reduction under general anesthesia and single-staged anteroposterior spinal reconstruction for fracture-dislocation of lower cervical spine. J Spinal Disord Tech 2015;28:E1-8.

40. Lins CC, Prado DT, Joaquim AF. Surgical treatment of traumatic cervical facet dislocation: anterior, posterior or combined approaches? Arq Neuropsiquiatr 2016;74:745-9.

41. McAfee PC, Bohlman HH, Ducker TB, et al. One stage anterior cervical decompression and posterior stabilization: a study of one hundred patients with a minimum of two years of follow up. J Bone Joint Surg (Am) 1995;77:1791-800.

42. Wang J, Chen H, Cao P, et al. Combined anterior-posterior fixation and fusion for completely dislocated hangman's fracture: a retrospective analysis of 11 cases. Clin Spine Surg 2017; 30:E1050-4.

43. Loher TJ, Bärlocher CB, Krauss JK. Dystonic movement disorders and spinal degenerative disease. Stereotact Funct Neurosurg 2006;84:1-11.

44. Harada T, Ebara S, Anwar MM, et al. The cervical spine in athetoid cerebral palsy. A radiological study of 180 patients. J Bone Joint Surg 1996;78:613-9.

45. Onari K, Kondo S, Mihara H, et al. Combined anterior-posterior fusion for cervical spondylotic myelopathy in patients with athetoid cerebral palsy. J Neurosurg 2002;97:13-9.

46. Galbusera F, Bassani T, Stucovitz E, et al. Surgical treatment of spinal disorders in Parkinson's disease. Eur Spine J 2018; 27:101-8.

47. Riew KD, Sethi NS, Devney J, et al. Complications of buttress plate stabilization of cervical corpectomy. Spine (Phila Pa 1976) 1999;24:2404-10.

48. Riew KD, Hilibrand AS, Palumbo MA, et al. Anterior cervical corpectomy in patients previously managed with a laminectomy: short-term complications. J Bone Joint Surg 1999; 81:950-7.

49. Joaquim AF, Makhni MC, Riew KD. Post-operative nerve injuries after cervical spine surgery. Int Orthop 2019;43:791-5.

50. Nassr A, Eck JC, Ponnappan RK, et al. The incidence of C5 palsy after multilevel cervical decompression procedures: a review of 750 consecutive cases. Spine (Phila Pa 1976) 2012; 37:174-8.

51. Riew KD. Rare complications of cervical spine surgery. Global Spine J 2017;7:6S.

52. Lee SH, Kim KT, Suk KS, et al. Effect of retropharyngeal steroid on prevertebral soft tissue swelling following anterior cervical discectomy and fusion: a prospective, randomized study. Spine (Phila Pa 1976) 2011;36:2286-92.

53. Lee SH, Mesfin A, Riew KD. Delayed esophageal perforation after anterior cervical fusion and retropharyngeal steroid use: a report of two cases. Spine J 2015;15:e75-80.

54. Fontes RBV, Kerolus MG, Kochanski RB, et al. Analysis and temporal evolution of extubation parameters for patients undergoing single-stage circumferential cervical spine surgery. Neurospine 2020;17:630-9. 